\title{
DIFICULTADES DE APRENDIZAJE DE LOS ESTUDIANTES UNIVERSITARIOS EN LA TEORÍA DEL CAMPO MAGNÉTICO Y ELECCIÓN DE LOS OBJETIVOS DE ENSEÑANZA
}

\author{
GUISASOLA, J., ALMUDÍ, J.M. y ZUBIMENDI, J.L. \\ Departamento de Física Aplicada I. Euskal Herriko Unibertsitatea
}

\begin{abstract}
Resumen. El objetivo principal de este trabajo es analizar las ideas que tienen los estudiantes sobre cuál es la naturaleza del campo magnético. Hemos supuesto que el conocimiento significativo de la fuente del campo magnético es un prerrequisito básico para que los estudiantes razonen sobre los fenómenos electromagnéticos. Para analizar las explicaciones de los estudiantes hemos tenido en cuenta que las personas construimos representaciones mentales para ayudarnos a comprender el funcionamiento de un sistema físico y que estas representaciones mentales incluyen diferentes categorías explicativas de la realidad en un mismo individuo dependiendo del contexto y del contenido que se trate. Assí pues, hemos diseñado un cuestionario de preguntas de tipo abierto con énfasis en las explicaciones, para analizar el razonamiento empleado por los estudiantes. Los resultados obtenidos parecen confirmar que los estudiantes presentan serias dificultades en el aprendizaje de la teoría del campo magnético.

Palabras clave. Magnetismo, dificultades de aprendizaje, categorías explicativas, progresión de aprendizaje, objetivos de enseñanza.

Summary. The aim of this study is to analyse students' ideas about the nature of magnetic field. We have assumed that a significant knowledge of the source of magnetic field is a basic prerequisite when students are to reason about electromagnetic phenomena. In order to analyse students' explanations, we have taken into account the fact that individuals build mental representations to help themselves understand the functioning of a physic system and that these mental representations include different explanatory categories of reality in one same individual depending on the context and the contents concerned. Therefore, we have designed an open-question questionnaire with an emphasis on explanations so as to analyse the students' reasoning. The results found seem to confirm that students do have serious difficulties in learning the theory of magnetic field.

Keywords. Magnetism, learning difficulties, learning progress, teaching aim.
\end{abstract}

\section{INTRODUCCIÓN}

Dentro del gran número de trabajos sobre concepciones de los estudiantes realizados durante los últimos veinte años, los temas más tratados han sido los considerados como más básicos dentro del marco teórico. En el área de física, se han tratado ampliamente temas de cinemática y dinámica (conceptos como velocidad, aceleración, fuerza...) dentro del campo de la mecánica (Carrascosa y Gil, 1992), así como los conceptos de intensidad de corriente eléctrica, voltaje y resistencia en circuitos sencillos de corriente continua en electricidad (Duit y von Rhöneck, 1998). Sin embargo, conceptos básicos de magnetismo han sido escasamente investigados en niveles de primaria (Barrow, 1987; Erickson, 1994; Bar et al., 1997) y secundaria (Maarouf y Benyamna, 1997; Seroglou et al., 1998; Borges y Gilbert, 1998; Borges, 1999). Recientemente han apare- cido algunos trabajos para nivel de universidad (Colombo y Fontdevila, 1990; Meneses y Caballero, 1995; Pais de Sousa, 1997; Galili y Kaplan, 1997; Bagno y Eylon, 1997; Velazco, 1998; Greca y Moreira, 1998). A continuación vamos a resumir brevemente, en el cuadro I, las principales aportaciones de algunos de los artículos mencionados, respecto a la identificación de las dificultades de aprendizaje en el electromagnetismo.

Varios de los trabajos citados muestran que el conocimiento de los estudiantes mejora con la edad y la instrucción, y que sus modelos mentales sobre los fenómenos electromagnéticos van evolucionando, siendo cada vez más complejos y acordes con los modelos científicos, pero no es menos cierto que, incluso después de un largo período de 
Cuadro I

Resumen de algunas de las investigaciones más relevantes.

\begin{tabular}{|c|c|}
\hline $\begin{array}{l}\text { Autor/es } \\
\text { Año } \\
\text { Nivel }\end{array}$ & Dificultades de aprendizaje \\
\hline $\begin{array}{l}\text { Colombo } \\
1990 \\
\text { Universidad }\end{array}$ & $\begin{array}{l}\text { Consideran que las dificultades de aprendizaje que se presentan en la enseñanza habitual son debidas fundamentalmente a la } \\
\text { forma de estructurar los contenidos en electromagnetismo. Proponen un programa, basado en la teoría de Ausubel, que utiliza } \\
\text { como concepto estructurador la energía y como subsunsor fundamental el concepto de corriente eléctrica. }\end{array}$ \\
\hline $\begin{array}{l}\text { Erickson } \\
1994 \\
\text { Primaria }\end{array}$ & $\begin{array}{l}\text { Analiza la interacción entre conocimiento de contenidos y tácticas de investigación, y para ello el autor utiliza el trabajo de } \\
\text { Karen Meyer, donde se da cuenta de los modelos explicativos del magnetismo (a través de imanes) de estudiantes de } 9 \text { a } 14 \\
\text { años, constatando los siguientes modelos: «tirar de», «efluvio» y «encerrar a». }\end{array}$ \\
\hline $\begin{array}{l}\text { Meneses } \\
\text { y Caballero } \\
1995 \\
\text { Universidad }\end{array}$ & $\begin{array}{l}\text { Concluyen que la mayoría de los estudiantes universitarios de Magisterio piensan que: } a \text { ) la fuerza magnética es una fuerza } \\
\text { central; } b \text { ) la fuerza magnética actúa sobre cargas en reposo; } c \text { ) confunden la fuerza magnética y el campo magnético, } d \text { ) } \\
\text { confunden los campos eléctrico y el magnético. }\end{array}$ \\
\hline $\begin{array}{l}\text { Bar, Zinn y Rubin } \\
1997 \\
\text { Secundaria }\end{array}$ & $\begin{array}{l}\text { Estudian las preconcepciones de los estudiantes sobre la interacción magnética y la gravitatoria. Concluyen que la mayoría } \\
\text { de los estudiantes piensa que para que se produzcan ambas interacciones es necesario un medio material. En el caso de la } \\
\text { gravedad, el aire; y en el caso de la fuerza magnética, un medio «conductor» similar al de la atmósfera terrestre. }\end{array}$ \\
\hline $\begin{array}{l}\text { Maarouf } \\
\text { y Benyamna } \\
1997 \\
\text { Secundaria }\end{array}$ & $\begin{array}{l}\text { Estudian las concepciones de los estudiantes frente a cuatro fenómenos relacionados con la interacción magnética. La } \\
\text { mayoría de los estudiantes explica la fuerza magnética en términos de un agente mediador que va del cuerpo que la produce } \\
\text { al receptor. Atribuyen al magnetismo el estatus de una «sustancia» localizada en el entorno de los cuerpos que interaccionan. }\end{array}$ \\
\hline $\begin{array}{l}\text { Pais de Sousa } \\
1997 \\
\text { Universidad }\end{array}$ & $\begin{array}{l}\text { En su trabajo de tesis doctoral concluye que los estudiantes piensan que: } a \text { ) la fuerza magnética actúa sobre las cargas en } \\
\text { reposo; } b \text { ) un hilo de corriente está cargado electrostáticamente; } c \text { ) las fuerzas magnéticas son centrales; } d \text { ) no explican la } \\
\text { interacción de un imán sobre un material de hierro. }\end{array}$ \\
\hline $\begin{array}{l}\text { Galili y Kaplan } \\
1997 \\
\text { Universidad }\end{array}$ & $\begin{array}{l}\text { Se plantean una serie de cuestiones acerca de la velocidad } v \text { que aparece en la ley de Lorentz y concluyen que la dependencia } \\
\text { de la fuerza con la velocidad tal y como se presenta habitualmente en clase contribuye a la fragmentación del conocimiento } \\
\text { de la física. Proponen una enfoque relativista «cualitativo» como base para presentar el electromagnetismo en cursos } \\
\text { introductorios de física en último curso de secundaria y primero de universidad. }\end{array}$ \\
\hline $\begin{array}{l}\text { Bagno y Eylon } \\
1997 \\
\text { Universidad }\end{array}$ & $\begin{array}{l}\text { En la primera parte del trabajo, investigan sobre las ideas centrales que tiene estudiantes de primer curso de universidad en } \\
\text { el ámbito del electromagnetismo. Concluyen que la mayoría posee representaciones memorísticas de ecuaciones que no } \\
\text { siempre son las más representativas. Así, indican que la ecuación que más se menciona es la ley de Ohm y que son muy } \\
\text { minoritarias las ecuaciones de Maxwell como representativas del electromagnetismo. }\end{array}$ \\
\hline $\begin{array}{l}\text { Borges y Gilbert } \\
1998 \\
\text { Borges, } 1999 \\
\text { Secundaria } \\
\text { Universidad }\end{array}$ & $\begin{array}{l}\text { Estudian los modelos mentales de estudiantes de secundaria, universidad y graduados sobre la interacción magnética. } \\
\text { Concluyen que se pueden identificar cinco modelos mentales: } a \text { ) el magnetismo como algo «que ejerce fuerza»; } b \text { ) magnetismo } \\
\text { como una «nube» que interacciona; } c \text { ) magnetismo como «electricidad»; } d \text { ) magnetismo como un fenómeno de polarización } \\
\text { eléctrica; y } e \text { ) magnetismo como modelo de campo. }\end{array}$ \\
\hline $\begin{array}{l}\text { Velazco } \\
1998 \\
\text { Universidad }\end{array}$ & $\begin{array}{l}\text { En su tesis de maestría encuentra que la mayoría de los estudiantes manifiesta cierta tendencia a otorgar realidad material a } \\
\text { entidades abstractas. Así, concibe la carga como un fluido, el campo como una sustancia y las líneas de campo como curvas } \\
\text { o trayectorias materiales. }\end{array}$ \\
\hline $\begin{array}{l}\text { Greca y Moreira } \\
1998 \\
\text { Universidad }\end{array}$ & $\begin{array}{l}\text { Realizan una categorización de las explicaciones de estudiantes cuando resuelven problemas y cuestiones de } \\
\text { electromagnetismo. Establecen cuatro categorías para el concepto de campo electromagnético: } a \text { ) no hay modelo físico del } \\
\text { campo; b) hay un modelo matemático que les permite resolver los problemas planteados; } c \text { ) existe algún tipo de modelo } \\
\text { mental físico para el campo; } d \text { ) construyen un modelo físico para el campo. Estos modelos evolucionan con la instrucción y } \\
\text { las personas pueden compartir varios hasta discriminarlos y adoptarlos de acuerdo con el contexto en que serán utilizados. }\end{array}$ \\
\hline
\end{tabular}

instrucción, la mayoría de los estudiantes no presenta un aprendizaje comprensivo de las nociones básicas de la teoría de campo electromagnético.

La gran mayoría de los trabajos citados se centran en analizar las concepciones de los estudiantes respecto a la fuerza magnética que se ejerce en diferentes fenómenos elec- tromagnéticos. En el nivel de primaria y menos en el nivel de secundaria, los trabajos se centran en aspectos particulares como el caso de las interacciones entre imanes. Sólo en alguno de los trabajos en el ámbito universitario se realiza un análisis más global que incluye no sólo la fuerza magnética sino también el campo magnético. Así pues, creemos que son necesarios más trabajos de investigación 
que intenten analizar las dificultades de los estudiantes en el aprendizaje del concepto de campo magnético y su fuente en el ámbito de secundaria y universitario.

El concepto de campo magnético y su fuente constituyen cuestiones básicas sin las que no se puede construir una teoría científica de los fenómenos electromagnéticos. Creemos, en consecuencia, que esta parte del programa de física es de especial interés por las siguientes razones:

a) Es un dominio en el que intervienen bastantes conceptos diferentes (fuerza, velocidad, corriente eléctrica y campo) y los estudiantes encuentran muchas dificultades en analizar al mismo tiempo los diferentes conceptos, así como elegir aquéllos que son adecuados para explicar una situación concreta.

b) La existencia de dificultades de los estudiantes para identificar las fuentes del campo magnético, así como para relacionar las diversas fuentes del campo magnético como imanes y corrientes (Guisasola, Almudí y Ceberio, 1999).

c) La gran importancia que el concepto científico de campo magnético tiene en aplicaciones tecnológicas.

Una clara concepción de conceptos básicos como campo o fuentes del campo magnético, y del modelo unificador de los imanes y la corriente eléctrica como fuentes del campo magnético, es esencial si se quiere iniciar a los estudiantes en una visión científica de los fenómenos electromagnéticos. En física, el estudio del magnetismo es muy amplio e incluye diferentes temas como propiedades y producción del campo magnético, inducción magnética y radiación electromagnética. En este sentido, en los cursos universitarios de introducción al magnetismo se considera, en primer lugar, los casos más sencillos en donde el medio que rodea a las cargas móviles y corrientes eléctricas es el vacío. Además se supone que las corrientes consideradas son corrientes estacionarias, es decir, corrientes que permanecen constantes y no cambian en el tiempo. Así, en este estudio, cuando hablamos de campos magnéticos nos referiremos a campos magnéticos estacionarios producidos por cargas en movimiento con velocidad constante o, de forma equivalente, por corrientes estacionarias dentro de la teoría clásica del electromagnetismo definida por las ecuaciones de Maxwell. En nuestro estudio, consideramos los imanes en términos de materiales que en su interior poseen pequeñas espiras de corriente que producen un campo magnético externo, aunque sabemos que este modelo no es toda la teoría actualmente aceptada (Chabay y Sherwood, 1995). Por otra parte, el campo magnético parece producido por cargas móviles, ya que no se ha encontrado un monopolo magnético. Pero nosotros utilizamos el concepto de velocidad de la carga eléctrica desde el sistema de referencia en el que nos encontramos y desde ese sistema hablamos de fenómenos magnéticos. No hay que olvidar, pues, que los fenómenos magnéticos dependen del sistema de referencia del observador y que, en este sentido, son de naturaleza relativista.

De acuerdo con la revisión bibliográfica realizada y la relevancia del concepto de campo magnético y su fuente en el cuerpo teórico de la física, el problema que nos plantea- mos en este trabajo es responder a los siguientes interrogantes: ¿Cuáles son las concepciones de los estudiantes que se ponen de manifiesto cuando explican fenómenos magnetostáticos sencillos? ¿Se pueden agrupar estas concepciones en categorías explicativas e identificar una secuencia de progresión en el aprendizaje?

\section{MARCO TEÓRICO}

La investigación sobre las concepciones de los alumnos está centrando su atención en cómo aprende, progresa o cambia el estudiante en dominios específicos de conocimiento. La discusión sobre lo que significa comprensión, progresión en el aprendizaje o cambio conceptual es antigua y sigue teniendo mucha actualidad (Hashweh, 1986; Hewson y Thorley, 1989; Gruender y Tobin, 1991; Matthews, 1997).

Los modelos constructivistas de la década de los ochenta, basados inicialmente en el cambio conceptual, han puesto de relieve el conocimiento previo del estudiante y han evolucionado hacia modelos de cambio conceptual, procedimental y actitudinal (Duschl, 1990; Gil et al., 1999). Cada vez existe un mayor número de investigaciones, en la bibliografía, que estudian cómo comprenden los estudiantes a medida que progresa su aprendizaje, para poder elaborar hipótesis de progresión que faciliten la comprensión en cada dominio específico (Millar, 1996; Rahayun y Tytler, 1999). Estas hipótesis de progresión servirán para «esquematizar» la comprensión y para fundamentar desde el punto de vista psicológico la toma de decisiones en la secuenciación del contenido a enseñar (Adey, 1997).

En este trabajo, para estudiar la comprensión y progresión de los estudiantes en contenidos conceptuales relacionados con el campo magnético a nivel introductorio, hemos tenido en cuenta los trabajos mencionados en el párrafo anterior y que los procesos de aprendizaje pasan por estadios que se caracterizan como formas particulares de colaboración social (Vigotsky, 1978). La idea de Vigotsky de que la relación del individuo con el mundo no es una relación directa también juega un importante papel en la idea de «intelecto colectivo» como un sistema supraindividual de formas de pensamiento propuesta por Marton (1981) para establecer categorías explicativas de la realidad construidas por los individuos.

Marton (1981) en su abordaje fenomenológico nos habla de la existencia de «diferentes formas en las que las personas perciben y entienden la realidad» (p. 177) y cómo cada una de estas estructuras (conceptos y formas de razonamiento asociadas) se pueden considerar categorías de descripción de la realidad. Estas categorías pueden ser observadas en un gran número de individuos, de forma que el conjunto de estas representaciones denota un tipo de intelecto colectivo: «La misma categoría de descripción aparece en diferentes situaciones. El conjunto de categorías es, pues, estable y generalizable entre situaciones, incluso si los individuos se «mueven» de una categoría a otra en diferentes situaciones» (Marton, 1981, p. 195). Marton establece la distinción entre sujeto y objeto, resaltando que 
lo percibido depende del contenido, ya que «el significado de la tarea no depende sólo de la presentación y del orden en que se realiza sino también de la forma en que la cuestión se realiza y de determinados detalles de percepción» (Marton 1981, p.193). El autor sugiere que se puede utilizar esa idea de sistema supraindividual de formas de pensamiento como un instrumento válido para la descripción de cómo las personas piensan en una situación concreta y cuál es el peso que tiene en ese momento cada uno de los conceptos de esta representación mental colectiva.

En trabajos recientes, Mortimer $(1995,1996)$ muestra que las personas pueden tener diferentes formas para ver y representar una misma realidad. Hewson y Thorley (1989) hablan del «cambio de estatus» que la persona atribuye al nuevo concepto (científico) disminuyendo el del viejo concepto (cotidiano). En definitiva, creemos que es posible describir las ideas de los estudiantes a través de «categorías explicativas» (Marton, 1981) que nos permitan evaluar el aprendizaje como una evolución a través de una mayor cantidad de estadios intermedios que los dos tradicionales: concepción científica y concepción alternativa.

Así pues, en este trabajo nos proponemos como objetivos, en primer lugar, analizar las ideas de los estudiantes de primer ciclo de universidad y de último curso de secundaria sobre fenómenos magnetostáticos considerados básicos. En segundo lugar, discutiremos algunas implicaciones de lo anterior a la hora de elegir los objetivos de enseñanza en magnetostática.

\section{HIPÓTESIS REALIZADAS Y METODOLOGÍA UTILIZADA}

A la hora de identificar las concepciones de los estudiantes en magnetostática y teniendo en cuenta lo indicado en la introducción, encontramos razonable suponer que los estudiantes de último curso de secundaria y primer ciclo de universidad no hayan alcanzado un aprendizaje comprensivo de la naturaleza del campo magnético, así como de las interpretaciones científicas sobre fenómenos magnetostáticos básicos. En concreto, hemos aceptado como hipótesis de trabajo que los estudiantes presentarán confusión a la hora de identificar la fuente del campo magnético, que detentarán problemas al explicar las interacciones magnéticas y que no tendrán en cuenta la naturaleza relativista de dicho campo. Así mismo, y de acuerdo con Marton (1981), esperamos que las ideas detectadas no sean ideas aisladas sino que se estructuren en «categorías explicativas» que tengan una gradación respecto al modelo científico actual y que den una idea de la progresión del aprendizaje.

El diseño realizado para tratar de identificar las dificultades de los estudiantes al interpretar fenómenos relacionados con la naturaleza del campo magnético ha consistido en un cuestionario de 19 preguntas de tipo abierto con énfasis en las explicaciones. Las cuestiones propuestas hacen referencia a fenómenos magnéticos relacionados con conceptos tales como fuente del campo magnético (ítems de 1 a 9), interacción magnética (ítems 10 a 17) y natura- leza relativista (ítems 18 y 19) del magnetismo, los cuales son enseñados en clase durante un curso de introducción al electromagnetismo que sigue un programa de contenidos estándar apoyado en libros de física general como los de Tipler o Alonso-Finn. En el caso de los estudiantes de $2^{\circ}$ de bachillerato, el programa coincide con el oficial de la prueba de selectividad. La indagación sobre cada uno de los aspectos ha sido realizada diseñando más de un ítem, ya que contrastar el mismo problema en diferentes situaciones nos facilita el análisis de las características del conocimiento de los estudiantes (White y Gunstone, 1992). Los contenidos y objetivos de las cuestiones han sido validados por investigadores expertos en el campo del magnetismo y por profesores con experiencia en la investigación didáctica. Para ello se establecieron tres reuniones de trabajo. En la primera se presentó el cuestionario y se pidió a los tres profesores e investigadores en magnetismo que emitieran su opinión sobre la coherencia teórica de los ítems y de la resolución efectuada. Después de llegar a un acuerdo con respecto al enfoque teórico de las cuestiones y su resolución, se realizaron otras dos reuniones con otros tres profesores con experiencia en investigación educativa para estudiar la concordancia entre los ítems y sus objetivos. Se establecieron categorías de objetivos y se discutieron las posibles opciones hasta llegar a acuerdos. En aquellos casos en que no había consenso general (sólo dos ítems) se llegó al acuerdo por mayoría. Así mismo, se realizó un trabajo previo con pequeñas muestras de estudiantes (Guisasola, Almudí y Ceberio, 2001) que nos ha permitido realizar el actual cuestionario.

La muestra de este estudio está constituida por estudiantes de último curso de bachillerato $(\mathrm{N}=70), 1^{\circ}$ y $2^{\circ}$ de Ingeniería Técnica Industrial $(\mathrm{N}=65$ y $\mathrm{N}=60)$ y de $3^{\circ}$ de Ciencias Físicas $(\mathrm{N}=40)$. El cuestionario fue pasado por el mismo investigador a todos los grupos cuando los estudiantes habían terminado el estudio de los temas correspondientes al área de electromagnetismo. Todos los grupos habían recibido enseñanza sobre el tema de electromagnetismo en sus respectivos cursos, impartida por profesores competentes y con experiencia. Sin embargo, es preciso indicar que en el caso de los estudiantes de $2^{\circ}$ de Ingeniería, el programa del curso no contemplaba un marco teórico explícito de electromagnetismo, sino aplicaciones tecnológicas de las leyes de Maxwell al campo de la electrónica y la electricidad. Por otra parte, los estudiantes respondieron al cuestionario bajo condiciones de examen ( de 55 minutos. Como el cuestionario resultaba excesivamente largo para una sesión, se dividió en cuatro partes cuidando el equilibrio de la dificultad, y se repartió aleatoriamente. Hemos de indicar que, de forma general, los estudiantes no mostraron problemas para entender los enunciados de las cuestiones.

Los criterios de corrección del cuestionario han tenido en cuenta el marco teórico expuesto en el apartado anterior y se ha tratado de agrupar las explicaciones de los estudiantes en base a diferentes esquemas que reflejen las categorías utilizadas por los estudiantes al explicar los diferentes fenómenos magnéticos presentados. El análisis de los cuestionarios lo han realizado por separado los tres autores de este estudio en base a criterios de corrección previamente 
consensuados. Posteriormente, se han estudiado conjuntamente los resultados de cada análisis y se ha llegado a un alto grado de coincidencia en el diagnóstico de los resultados. Por razones de la necesaria brevedad de este artículo vamos a comentar 10 de los 19 ítems que componen los cuestionarios (Anexo).

\section{EXPLICACIONES DE LOS ESTUDIANTES SOBRE ALGUNOS FENÓMENOS MAGNÉTICOS}

En este apartado describiremos las concepciones de los estudiantes de último curso de bachillerato y primer ciclo de universidad después de la enseñanza del magnetismo, haciendo especial énfasis en las dificultades que permanecen a lo largo de la instrucción.

\section{¿Cómo explican los estudiantes la existencia del campo magnético en una zona del espacio?}

La primera parte del cuestionario contiene 9 preguntas para analizar las ideas de los estudiantes sobre las fuentes del campo magnético y la equivalencia de la corriente eléctrica y los imanes como fuentes del campo magnético. Las preguntas se han graduado de mayor a menor dificultad explicativa. Aquí presentaremos los resultados de los ítems $3,4,6,7$ y 8 (Anexo).

En los ítems 1 y 2, los estudiantes tienen que exponer su conocimiento declarativo de la fuente del campo magnético sin necesidad de aplicarlo a un contexto determinado. El número de respuestas correctas confirma que la mayoría de los estudiantes (alrededor del $60 \%$ de la explicaciones en los tres primeros niveles y del $80 \%$ para $3^{\circ}$ de Físicas) conocen que las cargas eléctricas en movimiento son fuentes del campo magnético. Sin embargo, cuando se aumenta la dificultad explicativa de la pregunta como ocurre en los ítems 3 y 4, donde los estudiantes tienen que aplicar su conocimiento sobre la fuente del campo magnético, el número de respuestas correctas disminuye (Tabla I).
Cuando los estudiantes tienen que aplicar sus conocimientos se constata una diversificación de los argumentos pero que coinciden en considerar como fuente del campo magnético las cargas en reposo. Así pues, las explicaciones de los estudiantes confunden las fuentes del campo eléctrico y el magnético. Por ello esta categoría se ha llamado eléctrica. Ejemplos de esta categoría de respuesta serían los siguientes:

«La aguja de una brújula se trata de una aguja imantada que se desvía por campos magnéticos. En este caso hay dos, el de la Tierra y el de la carga; por lo tanto, estará entre el $N$ y la carga.» (Estudiante de $2^{\circ}$ de EUITI, ítem 3 )

«Sí, porque la barra cargada crea un campo magnético en sus proximidades que ejercerá una fuerza que hará moverse a la brújula.» ( $3^{\circ}$ de Físicas, ítem 4$)$

Los porcentajes de respuestas para la categoría «eléctrica» son similares en los dos ítems y son convergentes con el porcentaje obtenido para el ítem 5, cuyo objetivo es, al igual que los anteriores, ver si los estudiantes identifican las cargas en movimiento como fuente del campo magnético.

Es preocupante el bajo porcentaje de respuestas correctas para estudiantes universitarios, incluidos los de $3^{\circ}$ de Físicas (alrededor del 50\%). Dentro del bajo nivel de respuestas correctas destaca el de $2^{\circ}$ de Ingenieros, que puede ser debido a que durante este curso no se contempla, en el currículo, la teoría del electromagnetismo sino aplicaciones tecnológicas derivadas de la misma. Esto implicaría que el aprendizaje realizado en los cursos anteriores ha sido memorístico y que el olvido de ecuaciones y leyes hace que los estudiantes razonen en base a concepciones alternativas no modificadas por la instrucción.

Los ítems 6 a 9 tienen como objetivo detectar qué ideas y razonamientos utilizan los estudiantes para justificar que los imanes generan campos magnéticos. Para los niveles de enseñanza implicados se ha considerado como justificación correctamente fundamentada aquélla que utiliza el modelo explicativo de Ampère para un imán como fuente

Porcentaje de respuestas de los estudiantes a las cuestiones 3 y 4 .

\begin{tabular}{|l|c|c|c|c|c|c|c|c|}
\hline & \multicolumn{4}{|c|}{$\begin{array}{c}\text { Cuestión 3 } \\
\text { Categorías }\end{array}$} & \multicolumn{4}{c|}{$\begin{array}{c}\text { Cuestión 4 } \\
\text { Categorías }\end{array}$} \\
\hline & Correcta & Eléctrica & Incodificable & No contesta & Correcta & Eléctrica & Incodificable & No contesta \\
\hline $2^{\circ}$ Bach. & 33 & 51 & 2 & 14 & 22 & 63 & 7 & 8 \\
\hline $1^{\circ}$ Ing. & 21 & 56 & 10 & 13 & 20 & 68 & 2 & 10 \\
\hline $2^{\circ}$ Ing. & 13 & 68 & 0 & 19 & 15 & 71 & 8 & 6 \\
\hline $3^{\circ}$ Físicas & 55 & 35 & 5 & 5 & 46 & 34 & 6 & 14 \\
\hline
\end{tabular}


Tabla II

Porcentaje de respuestas de los estudiantes a las cuestiones 6 y 7 .

\begin{tabular}{|l|c|c|c|c|c|c|c|}
\hline & \multicolumn{3}{|c|}{$\begin{array}{c}\text { Cuestión 6 } \\
\text { Categorías }\end{array}$} & \multicolumn{3}{c|}{$\begin{array}{c}\text { Cuestión 7 } \\
\text { Categorías }\end{array}$} \\
\hline & Ampère & Eléctrica & Líneas & Ampère & Eléctrica & Líneas & Naturaleza \\
\hline $2^{\circ}$ Bach. & 22 & 47 & 12 & 16 & 28 & 16 & 33 \\
\hline $1^{\circ}$ Ing. & 12 & 44 & 33 & 4 & 56 & 32 & 4 \\
\hline $2^{\circ}$ Ing. & 18 & 48 & 28 & 9 & 64 & 22 & 0 \\
\hline $3^{\circ}$ Físicas & 51 & 34 & 0 & 21 & 31 & 5 & 16 \\
\hline
\end{tabular}

Tabla III

Porcentaje de respuestas de los estudiantes a la cuestión 8.

\begin{tabular}{|c|c|c|c|c|c|}
\hline \multicolumn{6}{|c|}{$\begin{array}{l}\text { Cuestión } 8 \\
\text { Categorías }\end{array}$} \\
\hline & A (Correcta) & Eléctrica & $\mathrm{B}=\mathrm{F}$ & Incodificable & No contesta \\
\hline $2^{\circ}$ Bach. & 2 & 55 & 38 & 4 & 1 \\
\hline $1^{\circ}$ Ing. & 6 & 30 & 56 & 4 & 4 \\
\hline $2^{\circ}$ Ing. & 5 & 37 & 57 & 0 & 1 \\
\hline $3^{\circ}$ Físicas & 18 & 43 & 32 & 4 & 3 \\
\hline
\end{tabular}

de un campo magnético. Los resultados obtenidos para los ítems 6, 7 y 8 (Anexo) se han agrupado en las tablas II y III. El número de respuestas incodificables o en blanco es muy minoritario, no superado por el $10 \%$ en los estudiantes universitarios.

En la categoría correcta «modelo de Ampère» se han agrupado aquellas explicaciones que describen el comportamiento de un imán según el modelo amperiano impartido en clase. La mayoría de estas explicaciones se fundamenta en que los electrones de los átomos que los componen, debido a su movimiento, generan dipolos magnéticos microscópicos que por estar orientados básicamente en la misma dirección se suman todos dando lugar a un dipolo magnético a nivel macroscópico que genera el campo magnético. Así mismo, en el ítem 8 se explica la relación de equivalencia entre espiras de corriente e imanes en base al movimiento de los electrones en el interior de los imanes. Sin embargo, el número de respuestas correctas se puede considerar muy bajo, si consideramos que el modelo teórico que justifica los imanes como fuente del campo magnético se ha impartido reiteradamente en los programas de estudio.
En la categoría «eléctrica», que es mayoritaria en las explicaciones de los estudiantes, se han agrupado los argumentos que hacen referencia a que el imán está cargado. Las cargas se reparten de manera que en el polo norte se acumula la carga positiva y en el sur la negativa, y después recurren a la analogía eléctrica para explicar los fenómenos magnéticos. Un ejemplo de este tipo de respuesta:

«Un imán es un elemento compuesto por dos partes, de las cuales una está cargada positivamente y la otra negativamente y eso genera un campo magnético de órbitas circulares que se mueven de polo a polo.» ( $1^{\circ}$ de EUITI, ítem 6$)$.

En esta categoría se produce una confusión entre los campos eléctrico y magnético atribuyéndoles a ambos la misma fuente, es decir, la carga eléctrica sin mencionar su estado de reposo o movimiento. Este tipo de confusión también se ha encontrado en otros trabajos sobre electromagnetismo (Meneses y Caballero, 1995; Salinas y Velazco, 1998; Borges, 1999).

Dentro de esta categoría eléctrica también se han incluido respuestas más elaboradas que la simple atracción y repul- 
sión, pero también basadas en la teoría eléctrica. Así, algunos estudiantes explican la atracción del clip por el imán (ítem 7) mediante la inducción eléctrica:

«Un clip es de metal, y los metales tienen en su estructura sus electrones libres. El campo generado por el imán polariza al imán y atrae o repele a los electrones, dependiendo del polo del imán que acerquemos. De esta manera, quedarán el polo positivo del imán con la zona negativa del clip, o viceversa, atrayéndose.» $\left(3^{\circ}\right.$ de Físicas, ítem 7)

Este tipo de explicaciones en base a la inducción eléctrica coincide con los resultados de otros trabajos, como los de Borges y Gilbert (1998), donde se constata que estudiantes y profesores utilizan un modelo de «polarización eléctrica» que explica que un imán actúa sobre los objetos bajo su influencia polarizándolos (en el sentido eléctrico del término).

Por otro lado, aparece un tipo de explicación alternativo al anterior que se basa en que los imanes producen campo magnético debido a que poseen líneas de campo. Este tipo de respuestas se han agrupado en la categoría «líneas de campo». Así mismo, parece que los estudiantes atribuyen «existencia real» a las líneas de campo desde una visión realista ingenua del campo magnético. Tipos de respuestas incluidos en esta categoría serían las siguientes:

«Un imán tiene dos polos, el $\mathrm{N}$ y el S. En éstos se generan líneas de campo, que salen del $\mathrm{N}$ y entran en el S. Dichas líneas crean un campo magnético alrededor del imán.» ( $2^{\circ}$ de bachillerato, ítem 6)

«Porque un imán tiene un sumidero donde se acumulan las líneas de campo y una fuente donde se producen, las cuales son las que crean el campo magnético.» ( $2^{\circ}$ de EUITI, ítem 6$)$

«El imán creará unas líneas de campo (que son el campo magnético), que actuarán sobre el clip atrayéndolo.» $\left(2^{\circ}\right.$ de bachillerato, ítem 7)

«Porque de los polos de un imán entran y salen líneas de campo; esas líneas de campo son líneas que llevan la dirección de $B$ y actúan sobre elementos que tengan electrones libres.» ( $2^{\circ}$ de Ingenieros, ítem 7)

Este tipo de explicaciones es convergente con los resultados de otros trabajos en los que se indica que los estudiantes tratan a las líneas de campo como entidades reales en el espacio en vez de utilizarlas como una propiedad vectorial del espacio (Törnkvist, Pettersson y Traströner, 1993; Pocovi y Finley, 1999).

Hay una minoría de respuestas que razonan en base a la «naturaleza propia» del material. Estas explicaciones indican que los imanes y el clip interaccionan (ítem 7) debido a su composición química (ferromagnética). Un ejemplo de este tipo de respuestas es el siguiente:

«Porque existen materiales como la magnetita capaces de generar campos magnéticos que crean fuerzas de atracción sobre metales como el hierro.» ( $1^{\circ}$ de Ingenieros, ítem 7$)$

Esta categoría explicativa también se encuentra en otros estudios realizados sobre la naturaleza de los imanes que encuentran explicaciones que atribuyen al magnetismo un estatus propio que depende del material (Bailey, Francis y
Hill, 1987). Este tipo de explicaciones disminuye drásticamente con la instrucción escolar y es muy minoritaria en niveles de educación superior (Maarouf y Benyamna, 1997).

Así mismo, en el ítem 8 existe un porcentaje importante de respuestas (entre el $32 \%$ y el $57 \%$ ) que presentan confusión entre el campo y la fuerza ejercida por éste. Así, en estas explicaciones se identifican dos conceptos que son epistemológicamente distintos, aunque relacionados entre sí como son el campo y la fuerza magnética. Por ejemplo:

«Sí están relacionados porque, en el primer caso, las corrientes inversas producen campos magnéticos opuestos que se repelen, y los imanes, por estar enfrentados por el mismo polo, producen campos magnéticos opuestos, y esto es lo que hace que se repelan.» (1 ${ }^{\circ}$ de Ingenieros, ítem 8$)$

Veremos que esta confusión aparece también de forma mayoritaria en las explicaciones sobre interacciones magnéticas en el siguiente apartado.

\section{¿Cómo interpretan los estudiantes las interacciones magnéticas?}

Los ítems 10 a 17 tienen como objetivo detectar las explicaciones de los estudiantes sobre fenómenos de interacción magnética. Los ítems 10, 12 y 17 presentan situaciones en las que se debe tener en cuenta que el campo magnético, al igual que el eléctrico y el gravitatorio, sólo actúa sobre su fuente, es decir, las cargas en movimiento. Así pues, se han considerado como correctas aquellas respuestas que explican que el campo magnético sólo actúa sobre cargas en movimiento y no lo hace sobre cargas en reposo. Los resultados obtenidos para estos ítems se describen en la tabla IV.

El porcentaje de respuestas incodificables o en blanco es muy pequeño (entre el 4\% y el 14\% según la cuestión y el curso) y nos indica el interés con que los estudiantes respondieron al cuestionario.

La gran mayoría de las respuestas (más de las tres cuartas partes de la muestra) indican erróneamente que existe interacción a pesar de encontrarse la carga en reposo y se han agrupado en la categoría B. Estas explicaciones que consideran que el campo magnético actúa sobre cargas en reposo también han sido detectadas en otros trabajos sobre magnetismo (Iowi, 1986; Meneses y Caballero, 1995; Pais de Sousa, 1997) en estudiantes de últimos cursos de secundaria.

La categoría $\mathrm{B}$ ha sido dividida en diferentes subcategorías según el agente que realizaba la interacción. Así, en la categoría B.1 (entre el 6\% y el 12\%) se han agrupado aquellas respuestas que atribuyen a las líneas de campo una entidad real y las identifican con la fuerza magnética. Ejemplos de este tipo de respuesta son:

«El imán posee un campo magnético y unas líneas de fuerza que entran por el sur y salen por el norte [hacen el dibujo], entonces, al ser la carga negativa, el imán las repele por las líneas de fuerza.» ( $2^{\circ}$ de Ingeniería, ítem 10$)$ 
Tabla IV

Porcentaje de respuestas de los estudiantes a las cuestiones 10, 12 y 17 .

\begin{tabular}{|l|c|c|c|c|c|c|c|c|c|c|c|c|}
\hline & \multicolumn{3}{|c|}{$\begin{array}{c}\text { Cuestión 10 } \\
\text { Categorías }\end{array}$} & \multicolumn{3}{c|}{$\begin{array}{c}\text { Cuestión 12 } \\
\text { Categorías }\end{array}$} \\
\hline & Correcta & $\begin{array}{c}\text { Líneas } \\
(\mathrm{B} .1)\end{array}$ & $\begin{array}{c}\text { Eléctrica } \\
(\mathrm{B} .2)\end{array}$ & $\begin{array}{c}\mathrm{B}=\mathrm{F} \\
(\mathrm{B} .3)\end{array}$ & Correcta & $\begin{array}{c}\text { Líneas } \\
(\mathrm{B} .1)\end{array}$ & $\begin{array}{c}\text { Eléctrica } \\
(\mathrm{B} .2)\end{array}$ & $\begin{array}{c}\mathrm{B}=\mathrm{F} \\
(\mathrm{B} .3)\end{array}$ & $\begin{array}{c}\text { Correcta } \\
\text { Categorías }\end{array}$ & $\begin{array}{c}\text { Líneas } \\
(\mathrm{B} .1)\end{array}$ & $\begin{array}{c}\text { Eléctrica } \\
(\mathrm{B} .2)\end{array}$ & $\begin{array}{c}\mathrm{B}=\mathrm{F} \\
(\mathrm{B} .3)\end{array}$ \\
\hline $2^{\mathrm{o}}$ Bach. & 12 & 12 & 53 & 11 & 20 & 6 & 34 & 24 & 8 & 6 & 59 & 13 \\
\hline $1^{\circ}$ Ing. & 4 & 4 & 76 & 12 & 15 & 0 & 50 & 26 & 20 & 0 & 53 & 15 \\
\hline $2^{\circ}$ Ing. & 6 & 11 & 66 & 3 & 7 & 3 & 71 & 13 & 6 & 11 & 63 & 11 \\
\hline $3^{\circ}$ Físicas & 48 & 9 & 31 & 0 & 46 & 0 & 32 & 8 & 46 & 9 & 37 & 0 \\
\hline
\end{tabular}

«Estoy en desacuerdo con la afirmación, ya que a un imán se le asocia un campo y también unas líneas de campo, las cuales van del polo norte al sur. Si la carga se encuentra en el campo del imán, éste tenderá a moverse en el mismo sentido que las líneas de campo y, por lo tanto, se pondrá en movimiento.» $\left(1^{\circ}\right.$ de Ingenieros, ítem 12)

«Yo creo que no, porque las líneas de campo efectuarían una acción [ponen el dibujo] de repulsa del imán uno y de atracción del imán dos; por lo tanto, la carga se dirigiría hacia el polo sur del imán dos.» ( $2^{\circ}$ de bachillerato, ítem 17$)$

«Se contrarrestan los flujos que proceden de un imán con el del otro, siempre que los imanes sean iguales y la distancia de la carga a ellos sea la misma.» ( $3^{\circ}$ de Físicas, ítem 17)

Este tipo de razonamientos para la explicación de la interacción magnética sería coherente con una idea de campo magnético asociada a líneas de campo con entidad real, que ya hemos comentado en el apartado anterior.

La mayoría de las respuestas se han agrupado en la categoría B.2, que hemos denominado eléctrica. La explicación que dan los estudiantes consiste en suponer que un imán o un campo magnético actúa sobre un cuerpo cargado que está en reposo, de la misma manera que lo harían dos cuerpos cargados estáticos, que interaccionan entre sí siguiendo la ley de Coulomb. Ejemplos de este tipo de respuesta son:

«La bolita es repelida, ya que, en un imán, las cargas negativas, electrones, salen del norte del imán y van al polo sur, de modo que el sur queda cargado negativamente, y así repelerá a la carga negativa.» ( $1^{\circ}$ de Ingeniería, ítem 10$)$

«Sí porque siempre habrá una atracción o repulsión; por lo tanto, un movimiento, dependiendo del signo de la carga y del polo que se le enfrente.» ( $3^{\circ}$ de Físicas, ítem 12$)$

Así mismo, casi todas las respuestas de esta categoría indican que los estudiantes consideran la interacción magnética como una fuerza central de igual dirección y sentido que la interacción eléctrica. Esto vendría a constatar que la identificación entre campo eléctrico y magnético no se produce sólo a nivel de la fuente sino también a nivel de la interacción, formando una explicación alternativa a la teoría científica explicada en clase. Así mismo, esta categoría de respuestas sería convergente con otros trabajos de investigación que explican las serias dificultades de los estudiantes para el análisis de fuerzas en el área del electromagnetismo (Galili, 1995; Pais de Sousa, 1997; Velazco, 1998). Veamos un ejemplo de este tipo de respuesta:

«No porque la carga $+Q$ positiva se ve atraída hacia el polo sur negativo del imán de la derecha y es repelida por el polo norte positivo del imán de la izquierda, hacia la derecha; de modo que la carga $+Q$ se desplazará hacia la derecha.» ( $1^{\circ}$ de Ingeniería, ítem 17)

Una minoría significativa de respuestas se ha agrupado en la categoría B.3. Estas respuestas confunden el campo magnético y la fuerza magnética, asignando el mismo vector para ambos. Este resultado es coherente con lo obtenido en el ítem 8 expuesto en el apartado anterior. Veamos algunos ejemplos:

«...que se producirá un $B$ y este campo del imán hará moverse al péndulo.» ( $2^{\circ}$ bachillerato, ítem 10$)$

«Si el campo magnético es suficientemente fuerte, tiende a mover la partícula cargada en la dirección del campo magnético o en la opuesta, dependiendo de si la carga es positiva o negativa.» ( $3^{\circ}$ de Físicas, ítem 12 )

«No se encontrará en equilibrio, ya que está sometido al campo magnético del imán de la izquierda y del de la derecha, que lo llevarán en una dirección.» ( $1^{\circ}$ de Ingenieros, ítem 17)

«Los dos imanes crean campos magnéticos que actúan sobre la carga, pero, como tienen sentidos opuestos, se contrarrestan.» $\left(2^{\circ}\right.$ de Ingenieros, ítem 17$)$

\section{¿Los estudiantes tienen en cuenta la naturaleza relativista del campo magnético?}

La última parte del cuestionario (ítems 18 y 19) tiene como objetivo constatar si los estudiantes conocen que el campo 
magnético depende del sistema de referencia elegido para medirlo. En el ítem 19, deben tener en cuenta el sistema de referencia desde el cual se analiza la existencia o no del campo magnético.

En la tabla V se muestran los resultados obtenidos agrupados en diferentes categorías. Se pueden distinguir dos grandes grupos, el primero contiene aquellas explicaciones que reconocen correctamente que la elección del sistema de referencia condiciona el análisis del fenómeno y su resultado (categoría A). Veamos algunos ejemplos de esta categoría:

«Sí, porque, aunque el campo se mide en el mismo sitio y en el mismo instante, el sistema de referencia puede ser distinto.» $\left(2^{\circ}\right.$ de bachillerato, ítem 19)

«Sí, porque para el alumno A1 sus cargas se podrían desplazar a diferente velocidad que para el alumno A2, por lo que el campo magnético sería diferente.» ( $2^{\circ}$ de EUITI, ítem 19$)$

El segundo grupo contiene aquellas explicaciones que no tienen en cuenta el sistema de referencia, atribuyendo, a factores irrelevantes como errores de medida, la diferencia de resultados (categoría B.1) o bien afirmando explícitamente que, si el fenómeno es el mismo y las circunstancias no cambian, el campo magnético debe ser único (categoría B.2). El número de respuestas en blanco aumenta respecto a otros ítems del cuestionario y nos indica la perplejidad de los estudiantes ante la situación planteada.

El número de respuestas correctas indica un pobre aprendizaje de la naturaleza relativista del campo magnético. Este resultado es convergente con los resultados del ítem 18 y con otros estudios que indican la importancia del sistema de referencia para analizar las semejanzas y diferencias entre el campo eléctrico y el magnético, así como para analizar la velocidad $v$ que aparece en la ley de Lorentz (Galili y Kaplan, 1997). Sin embargo, la mayoría de las respuestas de los estudiantes apoya una interpretación del campo magnético independiente del sistema de referencia desde el que se analiza el fenómeno y, por tanto, hablan de campo magnético en un punto del espacio de forma absoluta y única. Ejemplos de este tipo de respuestas son:
«No puede ser; dos sistemas medidos en las mismas condiciones no pueden crear campos diferentes. El campo depende de la velocidad de las partículas, de su carga, su dirección de movimiento, del medio por el que se mueven y de la distancia a la que se encuentran; si todo permanece igual, el campo también se mantendrá» $\left(1^{\circ}\right.$ de Ingenieros $)$

«No, el campo magnético depende de las cargas y de la distancia, pero, si estos datos son conocidos e iguales, el valor del campo magnético debería ser el mismo para ambos.» ( $3^{\circ}$ de Físicas)

«Podría ser cierto si las dos cargas se encontrasen a distintas distancias cuando lo calcula el alumno A1 que cuando lo hace el A2, ya que el campo magnético no sólo depende de las cargas sino también de las distancias a las que se calcula el campo.» $\left(2^{\circ}\right.$ de Ingenieros)

\section{CONCLUSIONES: CATEGORÍAS EXPLICATI- VAS DE LOS ESTUDIANTES SOBRE LA NA- TURALEZA DEL CAMPO MAGNÉTICO}

De acuerdo con los resultados obtenidos acerca de las interpretaciones de los estudiantes en fenómenos relacionados con la naturaleza y la fuente del campo magnético, podemos decir que la mayoría de los estudiantes posee un conocimiento declarativo adecuado (ítem 1 y 2) (Guisasola, Almudí y Ceberio, 1999, 2001). Sin embargo, cuando tienen que aplicar significativamente dicho conocimiento, una parte importante de los estudiantes se resiste a aceptar la existencia de una propiedad (el campo magnético) que no se manifiesta mediante un comportamiento perceptible; $y$, en la búsqueda no científica de esa evidencia, presentan dos modelos diferentes. Uno de los modelos construidos dentro de esta visión ingenua lleva a una minoría de estudiantes al extremo de atribuir la causa del magnetismo a «cualidades» de la propia materia en el caso de los imanes (ítem 7). El otro modelo explicativo, seguido por una parte significativa de estudiantes, atribuye, a las líneas de campo, existencia real (ítems 6 y 7). Esta visión de los fenómenos magnéticos hace que los estudiantes presenten un razonamiento «artificial» donde la visualización de las líneas de campo «obliga» a que exista un campo magnético; es decir, indican que la existencia del campo magnético es

Tabla V

Porcentaje de respuestas de los estudiantes a la cuestión 19.

\begin{tabular}{|l|c|c|c|c|c|}
\hline & \multicolumn{5}{|c|}{$\begin{array}{c}\text { Cuestión 19 } \\
\text { Categorías }\end{array}$} \\
\hline & A (Correcta) & B.1 & B.2 & Incodificable & No contesta \\
\hline $2^{\text {o }}$ Bach. & 6 & 44 & 32 & 11 & 7 \\
$1^{\circ}$ Ing. & 11 & 48 & 11 & 11 & 19 \\
$2^{\circ}$ Ing. & 6 & 27 & 37 & 15 & 15 \\
$3^{\circ}$ Físicas & 33 & 49 & 6 & 6 & 6 \\
\hline
\end{tabular}


debida a que existen las líneas de campo. Ahora bien, la secuencia de razonamiento correcta implica: a) considerar que la «visualización» de las líneas de campo es el efecto de algo; y b) analizar qué es lo que sucede para que se produzca esa «visualización».

Este modelo de líneas de campo «reales» también es utilizado por una parte significativa de los estudiantes para explicar las interacciones magnéticas (ítems 10 y 12). Explican que las interacciones entre imanes y cargas en movimiento o con otros imanes se producen debido a «repulsiones» o «atracciones» entre las líneas de campo, chocando entre ellas.

El segundo nivel de interpretación lo hemos denominado nivel eléctrico y se caracteriza por identificar la fuente del campo magnético con la carga eléctrica tanto en reposo como en movimiento. Los resultados parecen indicar que la mayoría de estudiantes (ítems 3, 4, 6 y 7) utiliza un modelo de analogía eléctrica que confunde el campo eléctrico y el magnético. Este modelo considera la interacción magnética como una fuerza central de tipo coulombiano y confunde el campo magnético con la fuerza magnética (ítems 10, 12 y 17). Otra consecuencia de esta categoría explicativa es que se justifica la naturaleza magnética de los imanes en base a que sus polos poseen cargas de signo opuesto (ítems 6,7 y 8 ).

Un cambio fundamental se produce en las explicaciones de los estudiantes cuando se distingue claramente entre el nivel macroscópico y el microscópico. Esto conduce a que no se confundan o superpongan el referente empírico de fenómenos magnéticos (la interacción magnética) con el contexto microscópico de interpretación (campo magnético). Este cambio diferencia las explicaciones de este nivel que hemos denominado amperiano respecto a las anteriores. Este nivel posibilita una correcta identificación de la fuente del campo magnético con las cargas en movimiento. Así mismo, en esta categoría explicativa se justifican las propiedades magnéticas de la materia en base al modelo de Ampère explicado en clase y se sabe justificar la relación entre imanes y espiras de corriente. Aunque el marco teórico que engloba las características mencionadas ha sido repetidamente presentado en los programas de física superados por los estudiantes, sólo una minoría ofrece explicaciones situadas en este nivel.

Así mismo, pocos estudiantes (alrededor del 10\% en Ingeniería y un tercio de los de $3^{\circ}$ de Físicas) tienen en cuenta el carácter relativo de cualquier movimiento $\mathrm{y}$, por tanto, la naturaleza relativista del campo magnético. En este caso, los estudiantes utilizan una secuencia de razonamiento que simplifica las posibles variables que intervienen en el problema «olvidando» que todo movimiento, en este caso el de las cargas con velocidad, depende del sistema de referencia utilizado. Esta forma de razonamiento que la investigación denomina reducción funcional (Viennot, 1992; Salinas et al., 1996; Furió et al., 2000) se caracteriza por la tendencia a no tener en cuenta todas las posibles variables que influyen en un problema. Se utilizaría como regla heurística la simplificación del sistema físico considerando el movimiento de las cargas como absoluto y, por tanto, la observación del campo magnético como única (ítem 19).
Los resultados obtenidos parecen indicar que las ideas de los estudiantes se pueden agrupar en lo que Marton (1981) denomina categorías explicativas, que contienen una estructura conceptual y formas de razonamiento concretas. En los resultados obtenidos hemos comprobado que los estudiantes pueden utilizar más de una «categoría explicativa», dependiendo del contexto en que debe ser utilizada; sin embargo, se puede representar un modelo de progresión conceptual en la comprensión del concepto de campo magnético (Fig. 1), que nos indique el estado de aprendizaje de los estudiantes de acuerdo con la frecuencia en que cada «categoría explicativa» es utilizada. En la figura 1 las categorías descritas van de menor a mayor cercanía con la teoría científica, dentro de un marco maxwelliano del electromagnetismo clásico.

\section{ALGUNAS IMPLICACIONES PARA LAELEC- CION DE OBJETIVOS EN LA ENSENAANZA DEL CAMPO MAGNETICO}

El análisis realizado parece indicar que la mayoría de los estudiantes presentan ideas de «sentido común» a la hora de interpretar fenómenos magnéticos. El modelo teórico consistente con la teoría magnética (categoría de Ampère) requiere determinadas características que son opuestas a las categorías previas mencionadas en el apartado anterior. Estas características son las siguientes:

1) Estar familiarizado a nivel macroscópico con fenómenos relacionados con las fuentes del campo magnético como, por ejemplo, que los imanes y las cargas en movimiento producen campos magnéticos.

2) Saber interpretar la acción del campo magnético a través del concepto de campo y de las líneas de campo. Así mismo, reconocer que el principio de superposición es un hecho de la naturaleza que también se cumple para el campo magnético.

3) Saber distinguir de forma significativa el campo eléctrico producido por cargas en reposo del campo magnético producido por cargas en movimiento. Esto implica que se conocen las analogías y sobre todo las diferencias entre el campo eléctrico coulombiano y el magnético estacionario. En concreto se reconoce:

a) La diferencia entre la fuente del campo eléctrico y el magnético. En concreto, se reconoce el comportamiento diferente de un hilo recorrido por una intensidad de corriente y ese mismo hilo cargado electrostáticamente.

b) Las características de las líneas de campo eléctrico y de campo magnético.

c) Las acciones centrales del campo eléctrico y la nocentralidad de la acción magnética.

d) El campo eléctrico actúa sobre las cargas independientemente de su estado de movimiento, y el campo magnético sólo actúa sobre cargas en movimiento. 
Figura 1

Progresión del aprendizaje de acuerdo con las diferentes categorías explicativas.

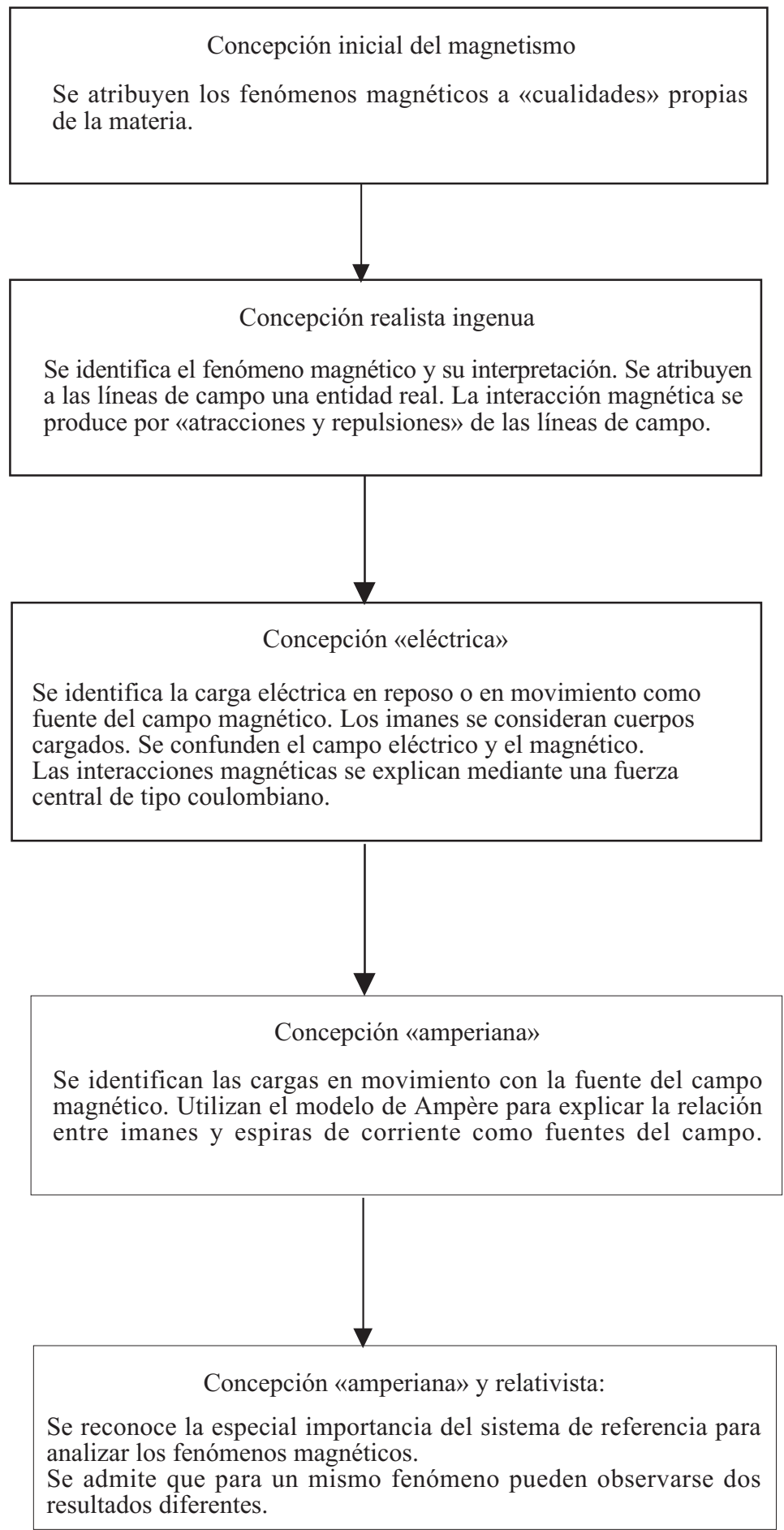

Incremento de la comprensión sobre los fenómenos magnéticos como diferenciación de los niveles macroscópicos y microscópicos. Se incrementa la diferenciación entre la interacción magnética y el campo magnético. Gradualmente se definen las cargas en movimiento como la fuente del campo magnético y se relacionan imanes y espiras de corriente.

Incremento de la comprensión sobre la naturaleza relativista de los fenómenos magnéticos. 
e) El diferente resultado de la ley de Gauss para el campo eléctrico y el magnético.

4) Superar la aparente dicotomía a nivel macroscópico entre las dos fuentes del campo magnético (imanes y corrientes) mediante la elaboración de un modelo científico a nivel microscópico que explique la naturaleza magnética de la materia.

5) Un objetivo final de una correcta interpretación de los fenómenos magnéticos sería reconocer el carácter relativista del campo magnético en el sentido de que, a diferencia de los otros campos de la naturaleza, como el gravitatorio y el eléctrico, en que sus fuentes son exclusivamente dependientes de la materia (masa y carga eléctrica respectivamente), en el caso del campo magnético, además de esto (carga eléctrica), depende del observador, a través de la velocidad de la carga creadora de dicho campo. Por lo tanto, será importante analizar con respecto a qué sistema de referencia se podrá medir esa velocidad.

Estos aspectos son un resumen de los principales objetivos que, de acuerdo con los resultados del estudio realizado, se deberían conseguir para que los estudiantes tuviesen una correcta comprensión de los aspectos conceptuales más importantes al finalizar un curso de introducción al magnetismo.

Ahora bien, en el análisis de las respuestas de los estudiantes hemos constatado razonamientos erróneos y dificultades procedimentales que es necesario abordar desde una enseñanza constructivista. Así pues, un buen conocimiento científico no puede reducirse sólo a aspectos conceptuales sino que debe haber, al lado de lo anterior, exigencias procedimentales y axiológicas a las cuales también habrá que referirse. Como indica Nerssesian (1995) «One plausible reason why physicists do not preach they practice is that for the expert, knowledge of thinking practices is largely tacit. Discussions of "tacit knowledge" usually focus on content knowledge, but knowledge of processes is equally important for the expert reasoner. As with riding a bicycle, once these are practised and facility has been developed, the processes become tacit.» (p. 204). En otras palabras, no se puede esperar que los estudiantes puedan asimilar todos estos contenidos conceptuales si no se tienen en cuenta aspectos procedimentales y ontológicos como serían los siguientes:

- Los estudiantes tienen que conocer cuáles son los problemas que justifican la introducción de nuevos conceptos; es decir, es necesario que los estudiantes perciban que los conceptos no se introducen de una manera arbitraria sino que son «invenciones» que se hacen con carácter ten- tativo y con el propósito de resolver situaciones problemáticas de interés, y que comprendan también la importancia de su tratamiento.

- Si queremos que los estudiantes vean la construcción de los conocimientos científicos como un proceso dinámico, que puede comportar cambios e incluso replanteamientos globales, consideramos necesario no presentar los conocimientos en su estado de elaboración final sino, más bien, realizar un proceso que lleve a construirlos.

- Además tendremos que elaborar una serie de actividades de manera que los estudiantes tengan la ocasión de utilizar las estrategias del trabajo científico como son: analizar la situación problemática que se presente, concebir hipótesis, diseñar y realizar experimentos, modelizarlos cuantitativamente (a través del lenguaje matemático y en su caso, gráfico), analizar los resultados obtenidos... De esta manera pueden comparar sus construcciones tentativas con las de la comunidad científica.

- Proponer actividades de reflexión y globalización, después de que se hayan analizado un «paquete» de ellas que atiendan a un objetivo común.

- Naturalmente un buen conocimiento supone la capacidad de utilizarlo en la resolución de todo tipo de problemas, en la interpretación de situaciones diversas, etc. En particular, la atención a situaciones problemáticas con interacciones CTS ha de ser un aspecto esencial en este y en cualquier campo científico, que permita salir al paso de incorrectas visiones descontextualizadas de la ciencia y que sirva para formar profesionales capaces de adoptar actitudes responsables ante el desarrollo científico y tecnológico y las implicaciones de todo tipo que éste conlleva.

Los aspectos indicados son importantes a la hora de diseñar la secuencia de enseñanza y para concretar de forma precisa la correspondencia entre objetivos y contenidos conceptuales. Por supuesto, estos objetivos, que tienen carácter general y, por lo tanto, válidos tanto para $2^{\circ}$ de bachillerato como para primer ciclo universitario, deberán concretarse en una serie de contenidos propios para cada nivel de enseñanza y, en consecuencia, con diferencias entre sí. Así pues, los profesores tendremos que elegir y secuenciar de forma justificada el programa de enseñanza a impartir; por consiguiente, el diseño y la elaboración de dicho programa - para cada uno de los niveles que han sido objeto de este trabajo- tendrá que ser coherente con las aportaciones de la investigación didáctica y de la epistemología científica, y tratará de superar las dificultades de aprendizaje detectadas; es este último aspecto el que será la meta de nuestros próximos trabajos. 


\section{REFERENCIAS BIBLIOGRÁFICAS}

ADEY, P. (1997). Dimensions of progression in a curriculum. The curriculum Journal, 8(3), pp. 367-390.

BAGNO, E. y EYLON, B. (1997). From problem solving to a knowledge structure: an example from the domain of electromagnetism. American Journal of Physics, 65(8), pp. 726-736.

BAILEY, J., FRANCIS, R. y HILL, D. (1987). Exploring ideas about magnets, Research in Science Education, 17, pp. 113116.

BAR, V., ZINN, B. y RUBIN, E. (1997). Children's ideas about action a distance. International Journal of Science Education, 19(10), pp. 1137-1157.

BARROW, L.H. (1987. Magnet concepts and elementary students» misconceptions, en Novak J. Procedings of the 2. Int. Seminar Misconceptions and Educational Strategies in Science and Mathematics, vol. III. Ithaca: Cornell University.

BORGES, A.T. (1999). Como evoluem os modelos mentais, Ensaio. Pesquisa em Educaçao em Cièncias, 1, pp. 85-125.

BORGES, A.T. y GILBERT, J.K. (1998). Models of Magnetism. International Journal of Science Education, 20(3), pp. 361378 .

CARRASCOSA, J. y GIL, D. (1992). Concepciones alternativas en mecánica. Enseñanza de las Ciencias, 10 (3), pp. 314-328.

CHABAY, R. y SHERWOOD, B. (1995). Electric \& Magnetic interactions. Nueva York: Wiley.

COLOMBO DE CUDMANI, L. y FONTDEVILA, P.A. (1990). Concepciones previas en el aprendizaje significativo del electromagnetismo. Enseñanza de las Ciencias, 8(3), pp. 215-222.

DUIT, R. y RHÖNECK, C. (1998). Learning and understanding key concepts of electricity, en Tiberghien, A., Jossem, L. y Barojas, J. (eds.). Connecting Research in Physics Education with Teacher Education, pp. 55-62 (I.C.P.E. book 1998).

DUSCHL, R.A. (1990). Restructuring science education, en Teacher College Press, Columbia University, Nueva York. Trad. cast. (1997). Renovar la Enseñanza de las Ciencias. Madrid: Narcea.

ERICKSON, G. (1994). Pupils' understanding magnetism in a practical assesment context: the relationship between content, process and progression, en Fensham P., Gunstone, R. y White, $\mathrm{R}$. The content of Science. Londres.

FURIÓ, C., CALATAYUD M.L., BÁRCENA S. y PADILLA D.H. (2000). Functional fixedness and functional reduction as common sense reasonings in chemical equilibrium and in geometry and polarity of molecules. Science Education, 84, pp. $545-565$.

GALILI, I. (1995). Mechanics background influences students' conceptions in electromagnetism, International Journal of Science Education, 17(3), pp. 371-387.

GALILI, I. y KAPLAN, D. (1997). Changing approach to teaching electromagnetism in a conceptually oriented introductory physics course. American Journal of Physics, 65(7), pp. 657667.
GIL, D. et al. (1999). ¿Puede hablarse de consenso constructivista en la educación científica? Enseñanza de las Ciencias, 17(3), pp. 503-512.

GRECA, I.M. y MOREIRA, M.A. (1998). Modelos mentales y aprendizaje de física en electricidad y magnetismo. Enseñanza de las Ciencias, 16(2), pp. 289-303.

GRUENDER, C.D. y TOBIN, K. (1991). Promise and Prospect. Science Education, 75(1), pp. 1-8.

GUISASOLA, J., ALMUDÍ, J.M. y CEBERIO, M. (1999). Students', ideas about the source of magnetic field. Proceedings of the Second International Conference of the European Science Education Research Association (ESERA), pp. 89-91

GUISASOLA, J., ALMUDÍ, J.M. y CEBERIO, M. (2001). Student's mental representations and the choice of teaching goals, en Pintó, R. y Surinach, S. (eds.). International Conference Physics Teacher Education Beyond 2000. Selected Contributions, París: Elsevier Editions.

HASHWEH, M.Z. (1986). Towards an explanation of conceptual change. European Journal of Science Education, 8(3), pp. 229-249.

HEWSON, P.W. y THORLEY, N.R. (1989). The conditions of conceptual change. International Journal of Science Education, 11, pp. 541-553.

IOWI, U.M.O. (1986). Student's Misconceptions about Conservation Principles and Fields. Research in Science and Technological Education, 4(2), pp. 127-137.

MAAROUF, A. y BENYAMNA, S. (1997). La construction des sciences physiques par les représentations et les erreurs: cas des phenomènes magnétiques. Didaskalia, 11, pp. 103-120.

MARTON, F. (1981). Phenomenography- Describing conceptions of the world around us. Instructional Science, 10, pp. 177-200.

MATTHEWS, M.R. (1997). Introductory Comments on Philosophy ans Constructivism in Science Education. Science Education, 6, pp. 5-14.

MENESES, J.A. y CABALLERO, M.C. (1995). Secuencia de enseñanza sobre el electromagnetismo. Enseñanza de las Ciencias, 13(1), pp. 36-45.

MILLAR, R. (1996). Investigation des élèves en science: una approche fondeé sur la connaisance. Didaskalia, 9, pp. 9-30.

MORTIMER, E.F. (1995). Conceptual Change or conceptual profile change? Science and Education, 4(3), pp. 265-287.

MORTIMER, E.F. (1996). Constructivismo, mudança conceptual e ensino de ciências: para onde vamos? Investigaçoes em Ensino de Ciências, 1, pp. 20-39.

NERSESSIAN, N.J. (1995). Should physicist preach what they practice? Science Education, 4, pp. 203-226.

PAIS DE SOUSA, M.G. (1997). «Forças e campos magnéticos». Tesis doctoral. Universidad de Aveiro.

POCOVI, M.C. y FINLEY, L. (2001). Historical evolution of the field view and textbook account. Actas V International History Philosophy and Science Teaching Conference. Como (Italia). 
RAHAYUN, S. y TYTLER, R. (1999). Progression in primary school childrens conceptions of burning: toward an understanding of the concept of substance. Research in Science Education, 29(3), pp. 295-312.

SALINAS, J. y VELAZCO, S. (1998). Modelos para el campo eléctrico en estudiantes universitarios a posteriori de la instrucción. Memorias SIEF (IV Simposio de Investigadores en Educación en Física), pp. 341-351. La Plata. Argentina.

SALINAS, J., CUDMANI, L.C. DE y PESA, M. (1996). Modos espontáneos de razonar: un análisis de su incidencia sobre el aprendizaje del conocimiento físico a nivel universitario básico. Enseñanza de las Ciencias 14(2), pp. 209-220.

SEROGLOU, F., PANAGIOTIS, K. y VASSILIS, T. (1998). History of Science and instructional desing: the case of electromagnetism. Science and Education, 7, pp. 261-280.
TÖRNKVIST, S., PETTERSON, K.A. y TRANSTRÖMER, G. (1993). Confusion by representation: On student's comprehension of the electric field concept. American Journal of Physics, 61(4), pp. 335-338.

VELAZCO, S. (1998). «El campo electromagnético en la enseñanza y el aprendizaje de la física». Tesina predoctoral. Universidad Nacional de Tucumán.

VIENNOT, L. (1996). Raisonner en physique. La part du sens commun. París: De Boeck Université.

VIGOTSKY, L.S. (1978). Mind in Society: the development of higher psycological process, en Cole, M., John-Steiner,V., Scribner, S. y Souberman, E. (eds.). Cambridge: Havard University Press.

WHITE, T.R. y GINSTONE, F.R. (1989). Metalearning and conceptual change. International Journal of Science Education, 11, pp. 577-586.

[Artículo recibido en junio de 2001 y aceptado en noviembre de 2001.] 


\section{ANEXO}

Algunas preguntas del cuestionario.

\section{1a. PARTE}

1) Como ya sabes, la masa es la causante de que aparezcan campos gravitatorios, ¿cuál es la causa de que se produzcan campos magnéticos? Pon dos ejemplos y explícalos.

2) Como ya sabes, la brújula es un buen detector de campos magnéticos, ya que en su presencia gira hasta orientarse en la dirección del mismo. Si tenemos una brújula y colocamos cerca de ella una carga en reposo, ¿girará la brújula?

3) La línea de trazos de la figura representa la dirección del campo magnético terrestre. En el punto $A$ hay una brújula y en el punto $B$ se encuentra una partícula en reposo cargada con una carga $Q$ positiva.

a) Dibuja sobre $A$ una flecha que represente la orientación de la aguja de la brújula.

b) Explica las razones de tu respuesta.

4) ¿Una barra cargada con una carga $Q$ hará girar una brújula colocada en sus proximidades?

Explica tu respuesta lo más detalladamente posible.

6) Un imán crea un campo magnético a su alrededor debido a:

a) Que en un imán hay una parte donde se acumula carga positiva y otra donde se acumula carga negativa.

b) Que dentro del imán existen corrientes de electrones que llevan a la creación de un campo magnético.

c) Otra respuesta. (Si optas por esta última, explícala detalladamente).

7) ¿Por qué crees que un imán atrae a un material de hierro como, por ejemplo, un clip?

8) Tenemos los siguientes dos sistemas: a) por un lado, dos espiras que en el instante $t=0$ están como indica la figura 1 . Si posteriormente hacemos pasar por cada una de ellas una intensidad de corriente $I$, en sentidos opuestos, se disponen como indica la figura $2 ; b$ ) el otro sistema estaría formado por dos imanes, tal como indica la figura 3.

a) ¿Ambos efectos repulsivos presentan alguna analogía entre sí?

b) Explica detalladamente el comportamiento observado en el sistema $a$ (pareja de espiras) y en el sistema $b$ (pareja de imanes).

Figura 1



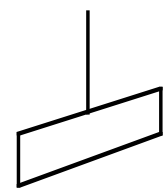

$\mathrm{F}_{\mathrm{r}}$



Figura 2
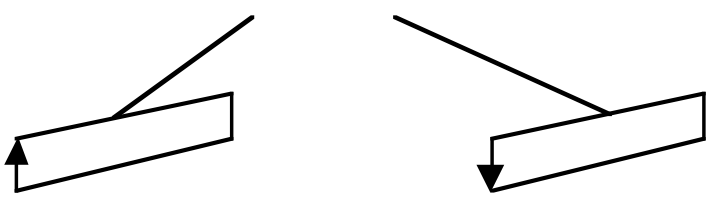

Figura 3

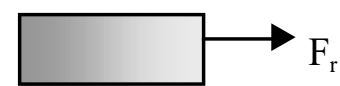


IIa. PARTE

10) Un estudiante afirma que en la situación de la figura 4, el imán y la bolita de poliestireno, cargada con una carga $Q$ negativa, se repelerán mutuamente. ¿Estás de acuerdo con dicha afirmación? Explícalo detalladamente

12) Explica si estás de acuerdo con la siguiente afirmación: «El efecto de un imán sobre una partícula cargada es ponerla en movimiento.»

17) ¿Se encuentra en equilibrio una partícula con carga $Q$ positiva, en la situación de la figura 5? Explica detalladamente tu respuesta.
Figura 4



Figura 5

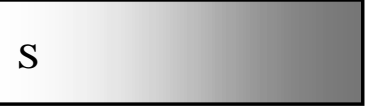

Q

$\mathrm{S} \quad \mathrm{N}$

\section{PARTE}

19) Un alumno A1 calcula el campo magnético total creado por dos partículas cargadas que se aproximan entre sí. Otro alumno A2 calcula el campo magnético creado por el mismo par de cargas (en el mismo lugar y en el mismo instante que el calculado por el alumno A1) y obtiene un resultado diferente. ¿Podría ser que los dos alumnos tuvieran razón? Explica detalladamente tu respuesta. 